\title{
Intelligent voltage regulator for distributed generation-based network
}

\author{
Zwawi Hamadouche ${ }^{1}$, Mounir Khiat ${ }^{2}$, Muhammad Asad Iqbal ${ }^{3}$ \\ ${ }^{1,2}$ Scamre Laboratory, Department of Electrical Engineering, National Polytechnic School of Oran-Maurice Audin \\ (ENPO-MA), Algeria \\ ${ }^{3}$ Department MS Electrical Engineering, Comsats University, Pakistan
}

\begin{tabular}{l} 
Article Info \\
\hline Article history: \\
Received Feb 8, 2021 \\
Revised Jun 8, 2021 \\
Accepted Jun 18, 2021 \\
\hline Keywords: \\
Artificial neural network \\
Distribution system \\
Solar panel \\
Voltage control \\
Wind turbine
\end{tabular}

\begin{abstract}
Power grids are being transformed into a smart distribution network that incorporates multiple distributed energy resources (DERs), ensuring stable operation and improved power quality at the same time. Many research papers have been published in recent years that discuss the voltage violation issues that emerge from the high penetration of distributed generation (DG). In this paper, we propose a new optimal voltage control technique based on feedforward neural networks (FFNN) to maintain a stable voltage profile. MATLAB $® /$ Simulink ${ }^{\circledR}$ has been used to carry out the simulation. The simulation results show the efficiency of this method in voltage control. The proposed approach ensured a stable voltage profile for the considered schemes.
\end{abstract}

This is an open access article under the $\underline{C C B Y-S A}$ license.

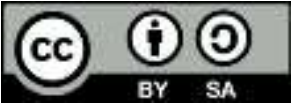

\section{Corresponding Author:}

Zwawi Hamadouche

Scamre Laboratory, Department of Electrical Engineering

National Polytechnic School of Oran-Maurice Audin (ENPO-MA), Algeria

Email: hamadouche92zouaoui@gmail.com

\section{INTRODUCTION}

The earlier electrical systems leverage a centralized model. In this model, the associated large power plants employ high power generators. The generators are interconnected to the underlying transmission network. Also, in this model, the transmission system is employed in the transportation of the generated energy that is usually from the central power plants to the respective consumers. It is noteworthy that the energy is usually transmitted over long distances. Based on this, high-voltage values are demanded for the effectiveness of the system. At the substations that are in close proximity to the consumption centers, the energy can be distributed via several lines with relatively lower-rated power and shorter length. In general, the conventional distribution system model exploits a power flow that is based on a one way from the transmission to the distribution network (DN) [1], [2].

Moreover, with current development, the existing centralized model concept has been reviewed with growing attention in the distributed model. This is in an effort to harness various energy resources in which small power plants can be deployed in a dispersed manner. This idea is typically referred to as a distributed generation (DG). It should be noted that the aforementioned energy resources are usually associated with renewable energies with a typical power rating lower than $50 \mathrm{MW}$ [1]-[3]. Furthermore, there are possibilities of DG unit connections to the system. This can be realized at different voltage levels that are usually from the low to high voltage. 
Additionally, the DG integration to the network changes the system control. Besides, it changes the quality of electrical power, resulting in certain constraints on the operation and functioning of the network [1]-[4]. This can be attributed to the fact that the new productions by the DG were not considered in the design of current systems [4]. In certain cases, the implementation can bring about an electric power quality indices deterioration. On the other hand, it can result in indices improvement. For instance, the alteration in the system can result in the occurrence of reactive energy flow, as well as the network and transformer overloads. Besides, the system is prone to over-voltages and subsequently, it results in power quality deterioration [5]. In contrast, deployment of the back-up units near enough to the center where they are demanded helps in saving the transmission power expense as well as the related transmission losses [1].

As aforementioned, there is a close correlation between DG and renewable energies. In this context, there has been a notable development in the wind and sun renewable energies. Also, their employment in the system presents a challenge not only regarding the efficient and safe operation but also on the underlying network control. To some extent, the issue can be addressed by means of microgrids. Microgrids are elements that manage resources in a distributed energy in a more dependable and decentralized way. Based on this, not only the control burden on the grid can be reduced but also their full benefits can be effectively exploited [1]-[6]. Moreover, depending on the generation and load type being deployed, microgrids can be ac or dc [7]. Also, power flow can be controlled in optimal manners between the microgrid and the public network using an appropriate interface. Besides, a smart grid has been presented to facilitate selfmanagement in the network [8].

Conceptually, a smart grid offers dynamic optimization using real-time measurements to enhance the system performance and ensure effective management in various aspects such as losses, security, and voltage levels. Based on the data gathered through the smart grid and its subsystems, the best approach to ensure suitable system operation by the system operators can be promptly identified [8], [9]. There are some efforts in which the insertion of DG energy producers into the DNs have been considered to analyze the system performance. One of such shows the benefits of DG installations for power generation in the distribution system. The work also presents notable enhancement that can be achieved in the distribution system's voltage profile as well as a reduction in the electric system losses [10]. Also, the effects of wind energy turbines connection on the voltage profile are considered in [11]. Besides, the load flow probabilistic technique is employed to study the related effects on voltage quality when wind turbines are interconnected with the DNs [11], [12]. Likewise, the analytical method implementations for influences of the wind power plant (WPP) on the system's reliability have been presented [12].

Moreover, voltage control can be independent-based and cooperative-based [13]. Likewise, a genetic code-based algorithm can also be employed for voltage regulation [14]. Also, voltage control can be achieved by employing an approach that is based on reactive power (RP) compensation. Similarly, a method that employed statistical analysis and can be used for regulating the voltage after the energy producer's insertion has been presented [15] Besides, an algorithm that can be employed for controlling the voltage using RP of energy producers has been presented [16]. Also, it should be noted that in a given network, the optimal sitting of the generators is imperative to improve the voltage profiles [17].

In this paper, we present a generalized model for a DN that is interconnected with a solar panel-type and wind turbine power generators to analyze the varied nature of the voltage. In addition, we consider effective means for optimal regulation by leveraging an artificial neural network (ANN) technique. Extensive simulations are carried out with MATLAB®/Simulink® to substantiate and support the presented control models. The rest of this paper is organized as follows: in Section 2, the employed mathematical models for electrical energy production with the main focus on the solar panel-type energy generator as well as a wind power producer are considered. Also, different development stages of intelligent voltage regulators for the PDEs are considered in Section 3. In Section 4, we present results and discussion. In Section 5, we present the concluding remarks.

\section{RESEARCH METHOD}

This section presents the employed mathematical models for electrical energy production. In this regard, we focus on the solar panel-type energy generator that is coupled to the grid via the inverter system and a wind power producer that is coupled to the grid using a synchronous generator. Also, we developed and modeled a typical configuration of these generators using MATLAB®/Simulink® platform. Furthermore, we present and explain a simplified diagram of a typical control for each generator.

\subsection{Wind turbine model}

The interaction between the wind rotor and wind is usually employed for representing the wind turbine aerodynamics. The features of this aerodynamic can be described using the disc theory. Based on the 
theory, the available wind power, $P v$, aimed at a particular disc swept by means of the rotor can be determined. Besides, the connection between the $P v$ and the extracted power from the rotor, $P_{t}$, is defined through the disc theory. Also, the instantaneous power, $P v$, can be expressed as [4]

$$
P v=\frac{1}{2} \rho a v^{3}
$$

where $\rho$ denotes the air density in $\mathrm{kg} / \mathrm{m}^{3}$, a represents the swept area by the rotor in $\mathrm{m}^{2}$, and $\mathrm{v}$ denotes the wind velocity in $\mathrm{m} / \mathrm{s}$. Also, the wind turbine's power coefficient is defined as (2)

$$
C p=\frac{P_{t}}{P_{v}}
$$

moreover, using (1) and (2), the extracted power from the rotor can be defined as [1]

$$
P_{t}=\frac{1}{2} \rho \pi r^{2} v^{3} C p
$$

where $\mathrm{r}$ denotes the rotor radius of the wind turbine in $\mathrm{m}$.

Also, the exerted rotational torque on the turbine by the wind can be expressed defined as (4)

$$
T_{M}=\frac{P_{t}}{\Omega}
$$

where $\boldsymbol{\Omega}$ represents the turbine rotor's angular mechanical velocity in $\mathrm{rad} / \mathrm{s}$. It should be noted that the aforementioned parameters are employed in the wind turbine modeling.

In general, the conventional system depends on the electricity delivery from the power producer plant to respective customers through the DN. This results in unidirectional flows. In contrast, the DG integration results in bidirectional flows of the associated active power (AP) in the DNs. Furthermore, in a scenario where the production surpasses the consumption, the flows can be toward the transport networks. This situation can present a considerable influence on the materials that are normally unidirectional such as equipment protection, and measurement devices, in the DNs. Moreover, a power flow reversal in the grid can be induced by the DG connection, resulting in bidirectional flows. Consequently, DG presents compatibility issue between the current network and the introduced energy. This demands modification of the existing electrical network protection plan [4].

Moreover, the DG integration can lead to a rise in the tension which can result in an overvoltage in the network. For instance, based on (2), for connections between a single power generator and the node, N, the voltage drop between the $\mathrm{N}$ connection point and the source station can be expressed as [4]

$$
\begin{aligned}
& \Delta U=\frac{R\left(P_{G}-P_{L}\right)+L_{\omega}\left( \pm Q_{G}-Q_{L} \pm Q_{C}\right)}{V_{N}} \\
& P_{N}=P_{G}-P_{L} \\
& Q_{N}= \pm Q_{G}-Q_{L} \pm Q_{C}
\end{aligned}
$$

where $P_{N}, P_{G}$, and $P_{L}$ are the AP at node N, AP supplied by the generator, and AP consumption, respectively, $Q_{N}, Q_{G}$, and $Q_{L}$ represent the RP at node N, RP supplied by the generator, and RP consumption, respectively, and $Q_{C}$ denotes the RP compensation device.

Moreover, to demonstrate the impact of the DG integration, we consider a scenario with a typical network that has $\mathrm{N}$ nodes and with connected $\mathrm{N}$ loads as depicted in Figure 1. During distribution, there will be voltage drops among the source and connection point, $\mathrm{Nj}$. The associated voltage drop can be expressed as [18].

$$
\Delta U\left(N_{j}\right)=\frac{\sum_{k=1}^{n}\left(\sum_{l=1}^{k<j} R_{l}\right) \cdot P_{k}+\sum_{k=1}^{n}\left(\sum_{l=1}^{k<j} X_{l}\right) \cdot Q_{k}}{U_{\text {source }}}
$$

where $\mathrm{P}_{\mathrm{j}}$ and $\mathrm{Q}_{\mathrm{j}}$ denote the AP and RP at the node $\mathrm{N}_{\mathrm{j}}$, Usource is the upstream voltage of the short-circuit impedance $\left(\mathrm{R}_{1} ; \mathrm{X}_{1}\right), \mathrm{j}=1,2, \ldots \mathrm{n}$, and $\mathrm{n}$ denotes the nodes. 


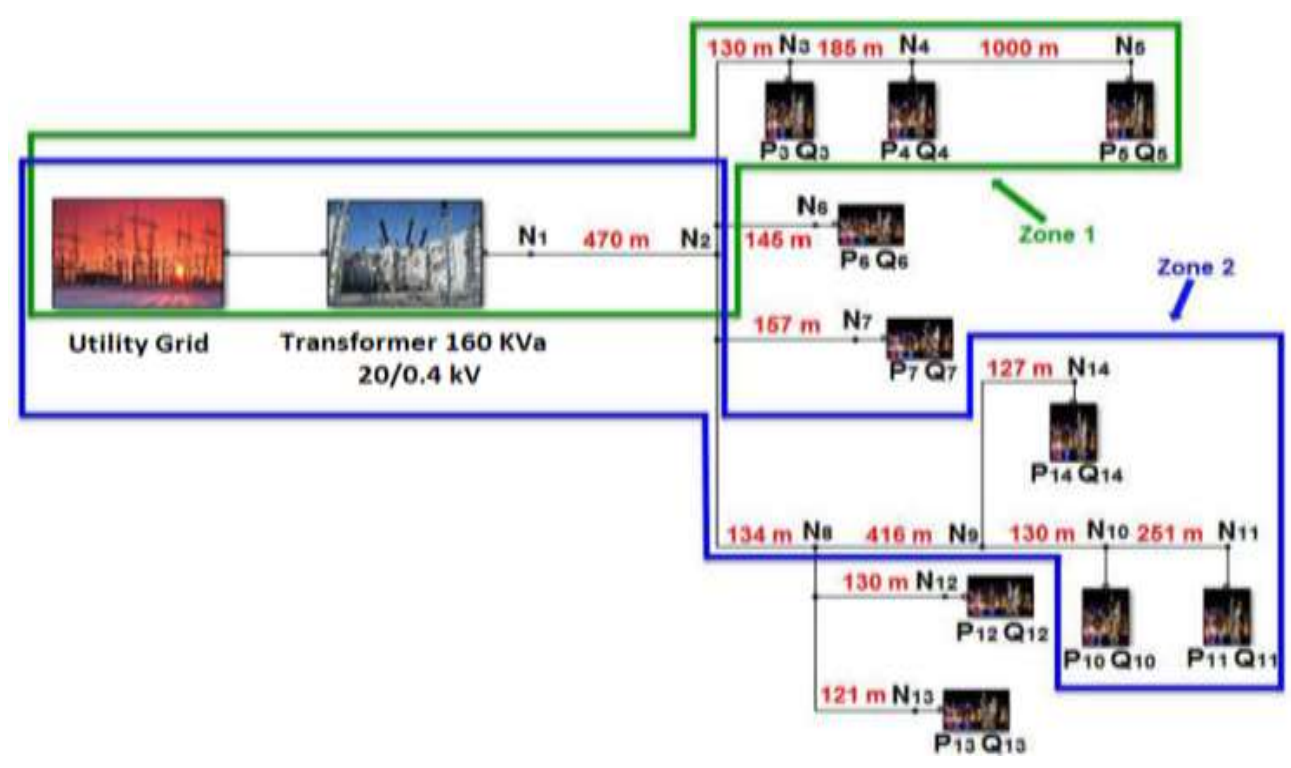

Figure 1. LV network without DG integration

\subsection{PV cell model}

The PV cell can be modeled using a diode, a photo current source, a parallel resistor, and a series resistor. In this context, the output current of the PV cell can be defined as [19], [20]

$$
I=I_{p h}-I_{d}\left[\exp \left(\frac{V+R_{S} I}{n V_{T}}\right)-1\right]-\frac{V+R_{S} I}{R_{S h}}
$$

where Iph is the short-circuit current due to sunlight (photons), Id denotes the shunted current through the diode, $\mathrm{V}$ denotes the voltage on the load, I is the current through the load, Rs and Rsh represents the parasitic series and shunt resistance of the array, respectively, and VT is given as [20]

$$
V_{T}=\frac{T_{c} k}{q}
$$

where $\mathrm{k}$ denotes the Boltzman's constant $(1.38 \times 10-23 \mathrm{~J} / \mathrm{K}), \mathrm{q}$ is the electron charge $(1.6 \times 10-19 \mathrm{C})$ and $\mathrm{T}_{\mathrm{c}}$ denotes the temperature of the operating module $\left({ }^{\circ} \mathrm{C}\right)[21]$.

Furthermore, the photocurrent Iph is a function of the solar irradiance received by the solar cell as well as its temperature and can be expressed as [19]-[22]

$$
I_{p h}=\left[I_{s c}+\alpha\left(T_{c}-T_{r e f}\right)\right] \cdot G
$$

where $\alpha=0: 0012 \times$ Isc denotes the temperature coefficient in $(\mathrm{A} / \mathrm{K})$, Isc is the short-circuit current, Tref represents at reference conditions $(298 \mathrm{~K})$ and $\mathrm{G}$ denotes the solar irradiance level $\left(\mathrm{kW} / \mathrm{m}^{2}\right)$.

Moreover, to develop a PV module, there is a need for solar cells. These are usually interconnected in series and parallel. With regards to a single-cell circuit module, the relationship between the PV module's voltage and current can be expressed as [19]

$$
I=N_{p} I_{p h}-N_{p} I_{d}\left[\frac{\exp \left\{q\left(\frac{V}{N_{S}}+\frac{I R_{S}}{N_{P}}\right)\right\}}{k T_{C} A}-1\right]-\frac{\frac{N_{p} V}{N_{S}}+I R_{S}}{R_{S h}}
$$

where $N_{s}$ and $N_{P}$ represent the solar cells within a module that are connected in series and parallel.

\subsection{Impact of the insertion of DG on the voltage profile}

In this subsection, we demonstrate the DG implementation impact using a 3-phase LV system. The system is modeled as well as simulated on the MATLAB®/Simulink® platform as illustrated in Figure 2. Also, we employ (8) to evaluate the voltage drop at the respective node. Furthermore, the network under consideration comprises 14 nodes and 10 loads ( $P$ and $Q)$. Moreover, a 160-kVA, 20=0:4-kV step-down 
transformer is used to supply the loads. We use this network as a case study due to the easy identification of the surge phenomenon. Besides, at the considered voltage level, the respective linear resistance is more imperative compared with the related linear reactance (i.e. R X). It should be noted that we consider two zones (i.e. 1 and 2) and the respective zone denotes a distribution line. Also, to demonstrate the DG impact, we consider scenarios without and with DG insertion.

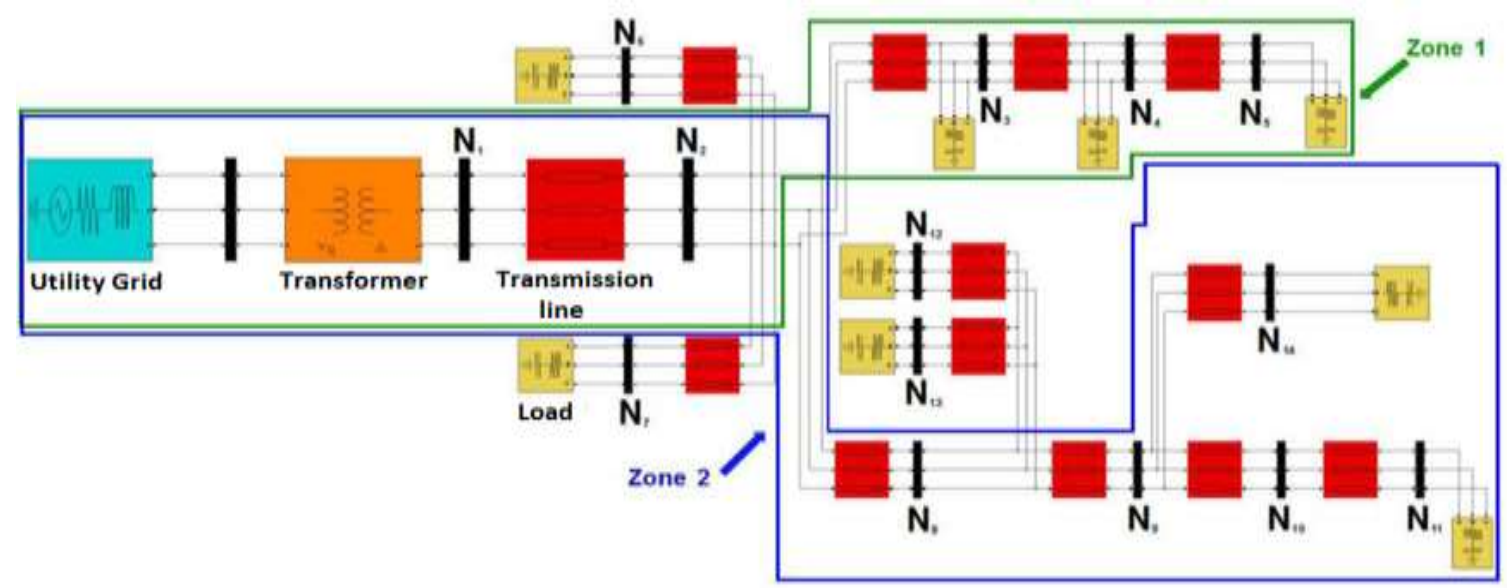

Figure 2. MATLAB®/Simulink® simulation of LV network without DG integration

a) Without DG: The voltage drop at the respective node is evaluated using the voltage profile without DG in Figure 2. In this scenario, $50 \%$ and $100 \%$ load states are considered. Since no DG is employed, the network is based on a conventional operation in which voltage drops occur between the source station and the consumption points. Simulation results presented in Figure 3 shows that for a full load (100\%) situation in both Zone 1 (3(a)) and Zone 2 (3(b)), the voltage gets to low values towards the end of the line.

b) With DG: In this part, we consider the insertion of a solar panel-type (node 11) and wind turbine (node 14) power generators into the network at zone 2 . The voltage drop at the individual node is evaluated using the voltage profile with DG in Figure 4. Moreover, half (50\%) and full (100\%) load states are considered in the simulation. The results presented in Figure 5 show that apart from the substantial surge in the voltage at the points where the DG is coupled, there is a notable increase across the neighboring nodes as well. Besides, regarding the half $(50 \%)$ load states, the voltage exceeds the acceptable voltage limit. The critical level is experienced from node 10 to node 14 as depicted in Figure 5(b) in Zone 2. The excess voltage is owing to the DG implementation and proportional to the delivered power. Consequently, the DG implementation can result in overvoltage on the voltage profile.
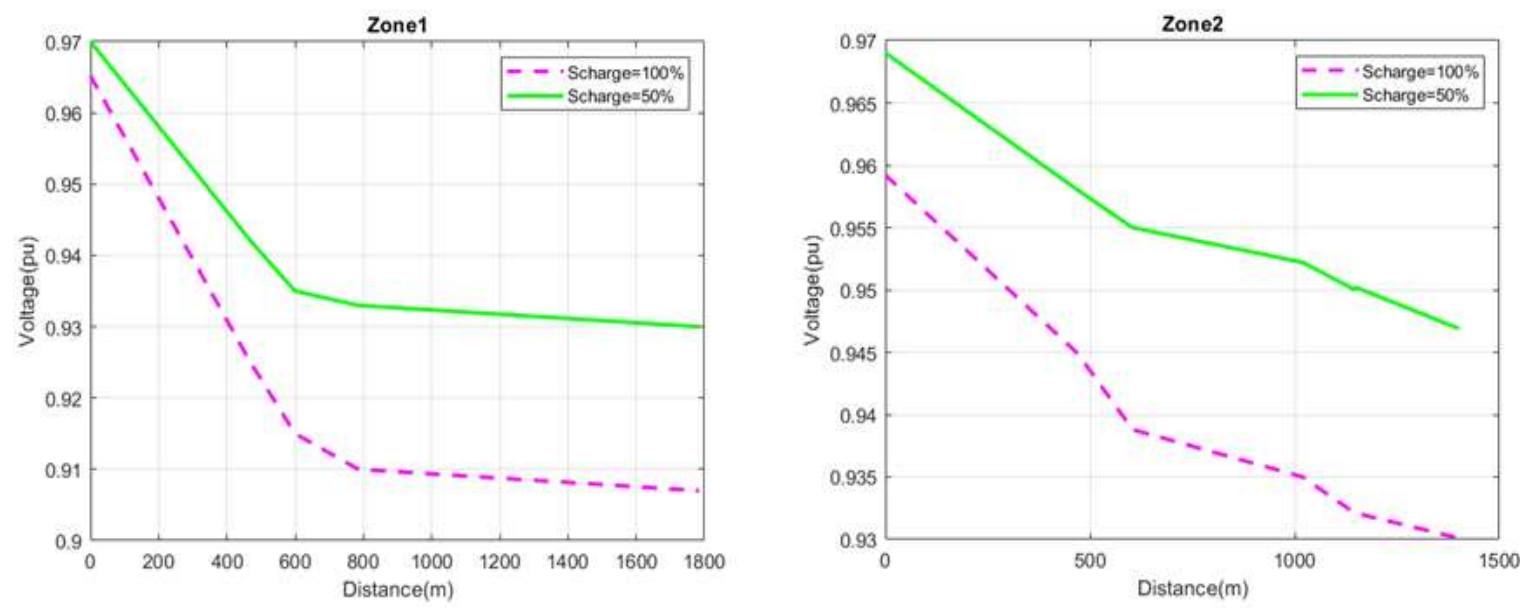

Figure 3. Voltage profile for 50\% and 100\% load states without DG; (a) zone 1 and (b) zone 2 


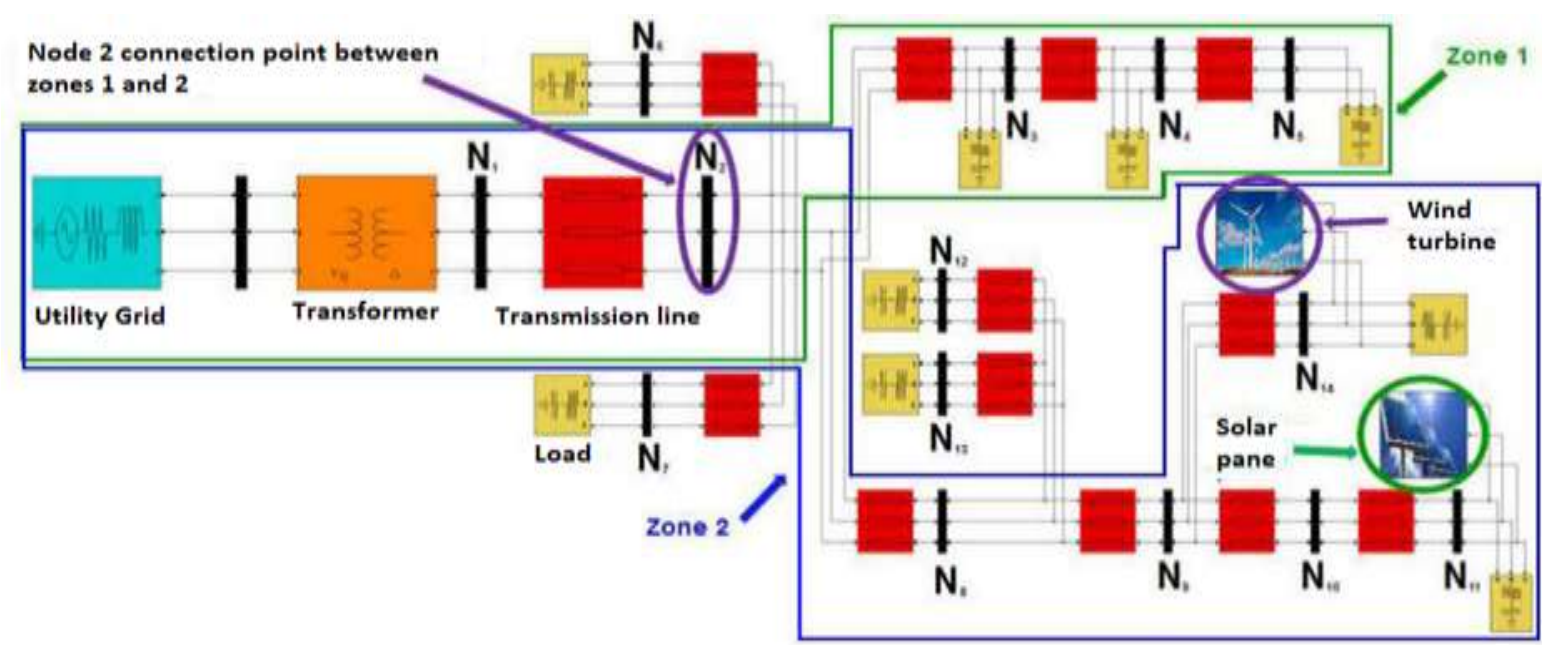

Figure 4. MATLAB $® /$ Simulink ${ }^{\circledR}$ simulation of LV network with integration of solar panel-type (node 11) and wind turbine (node 14) DG
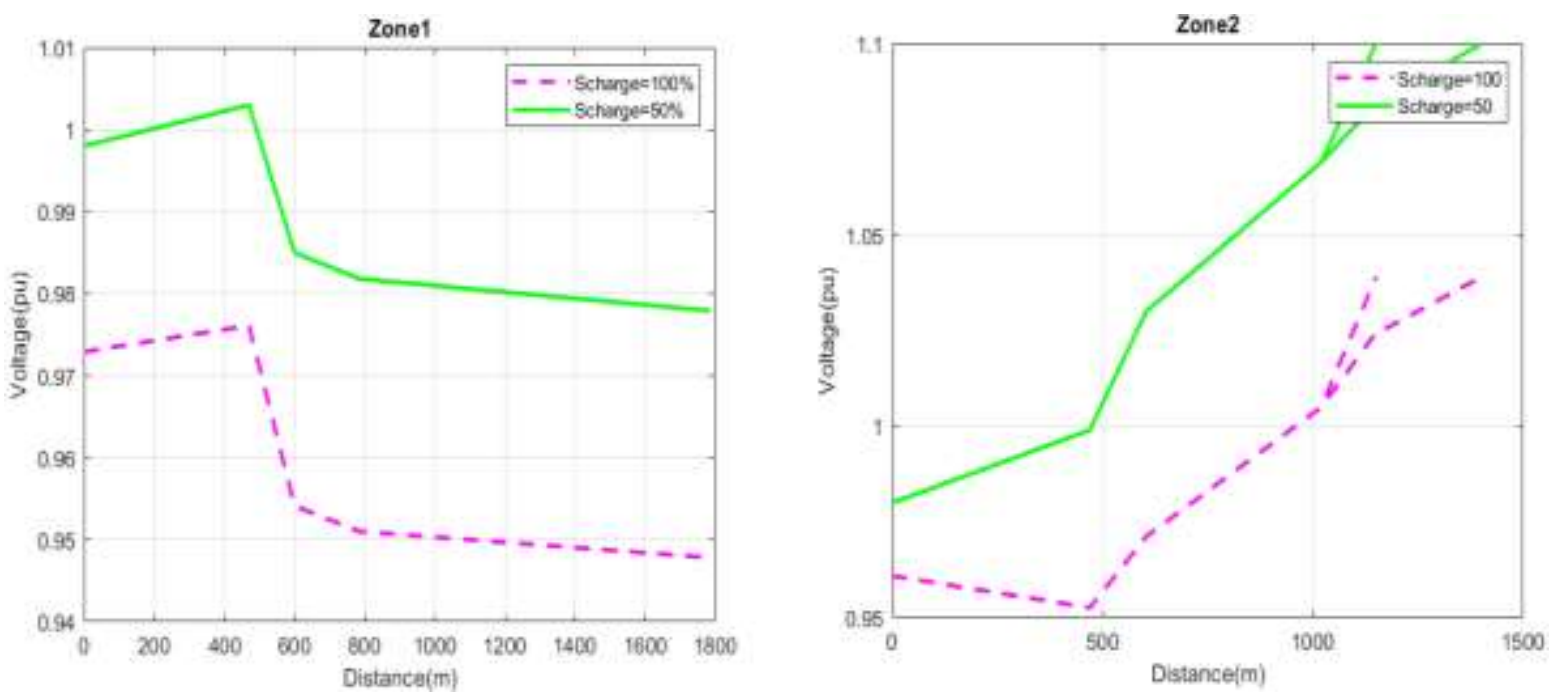

Figure 5. Voltage profile for 50\% and 100\% load states with DG; (a) zone 1 and (b) zone 2

\subsection{Intelligent voltage regulators for the pdes}

The section presents different development stages of intelligent voltage regulators for the PDEs.

\subsubsection{Artificial neural network (ANN)}

The 3-Phase LV system simulation presented in subsection 2.3 shows that the connection of DG units between loads can cause critical voltage regulation problems. Consequently, to offer a proper voltage regulation in the DN, we employ an ANN technique. An ANN is a biologically motivated computational related model [23]. It comprises neurons that are processing elements. The neurons are connected together and there are coefficients (weights) that are placed on the links. In this work, feedforward neural networks (FFNN) is employed because it is the most widely used model in a number of practical applications [24]. The schematic of FFNN with the backpropagation algorithm is depicted in Figure 6. An FFNN contains a chain of layers. For instance, it comprises different tiers such as the input, hidden, and output layers. The activation functions for computing the optimum weighting of input bounds regarding the target output values are contained in the hidden layers. Furthermore, to adapt weights and biases within the individual layer, a backpropagation algorithm is adopted for the training scheme. This is in an effort to lessen the errors between the network output and target value for the input-based factors [24], [25]. Moreover, the employed FFNN model comprises one input layer, two hidden layers, and one output layer. Also, the input as well as output 
layer dimensions are alike to the input and target parameter numbers. On the other hand, dimensions of the hidden layer are adapted manually with respect to the model performance [23]. The network model weights and associated biases are initialized by means of the MATLAB ® Neural Network Toolbox. Also, the model is updated through the configured functions such as adaption learning, training, activation, and performance. In addition, since the developed model is based on FFNN with the backpropagation algorithm, and the algorithm usually experiences not only a slow to converge but also an overfitting problem [23], a levenbergmarquardt (LM) regularization technique [25], [26] is employed to address the issues. This is due to its relatively swifter convergence in the backpropagation. Likewise, the "trainlm" training function is employed while the "mse" and "learngdm" in the toolbox are chosen for the performance functions and adaption learning, respectively. Besides, to achieve its output, any differentiable activation function can be employed by each neuron. The activation functions are located in the hidden layer and output layer neurons, but not in the input layer neurons.

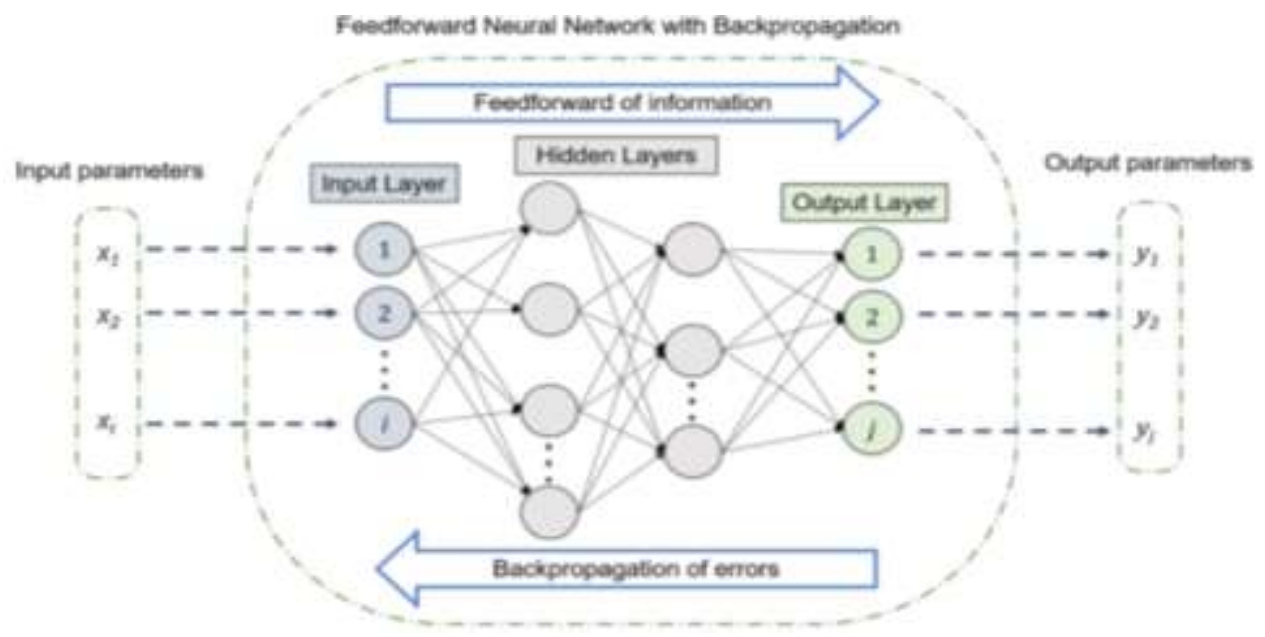

Figure 6. Schematic of feedforward neural networks with backpropagation algorithm

\subsubsection{Model performance assessment}

The respective model performance is evaluated by exploiting the mean-square error (MSE) and coefficient of determination (R2) that can be defined, respectively as [23]-[27]

$$
\begin{aligned}
& M S E=\frac{1}{n} \sum_{i=1}^{n}\left(\widehat{y}_{l}-\overline{y_{l}}\right)^{2} \\
& R^{2}=\frac{\sum_{i=1}^{n}\left(\widehat{y_{l}}-\hat{y}\right)^{2}}{\sum_{i=1}^{n}\left(y_{i}-\hat{y}\right)^{2}}
\end{aligned}
$$

where $\widehat{y}_{\iota}$ represents the predicted output using the neural network, $\bar{y}_{\iota}$ represents the mean of target values, $y_{i}$ denotes the obtained target value from data sets, and $\mathrm{n}$ represents the number of simulated scenarios.

\section{RESULTS AND DISCUSSION}

The results obtained show that the voltage control is effective since the setpoint values are indeed imposed on the connection node of the energy producers as shown in Figure 7. In addition, it is evident that there is a good correspondence between the excitation and generator voltages. Also, as shown in Figure 8, there is a noticeable correlation between the electric powers and the control voltage.

The MATLAB neural network toolkit is employed for neural network training and learning. The training state diagram of the backpropagation neural network is shown in Figure 9. The figure illustrates that the model reaches the convergence precision at step 101. Moreover, the trained neural network is then employed for prediction and verification. The validation performances for the solar power inverter model is presented in Figure 10. The most excellent validation performance is realized at the epoch 101 with a corresponding MSE value of 0:00038667. It is noteworthy that the validation, train, and test curves are relatively comparable. Also, it should be noted that the plot does not increase after convergence, verifying the stability of the model. This 
implies that there is no overfitting issue in the system by the ANN and that the adopted network training is efficient. Also, the error histogram for forecasting the voltage regulation with the developed ANN is represented in Figure 11. This plot emphasizes that most of the errors fall between $-1: 04454$ and 0:1193, which is a narrow interval. Also, the majority of the errors have a value of about 0:03736. Consequently, the error histogram illustrates that the forecasting accuracy of our proposed model is very good.
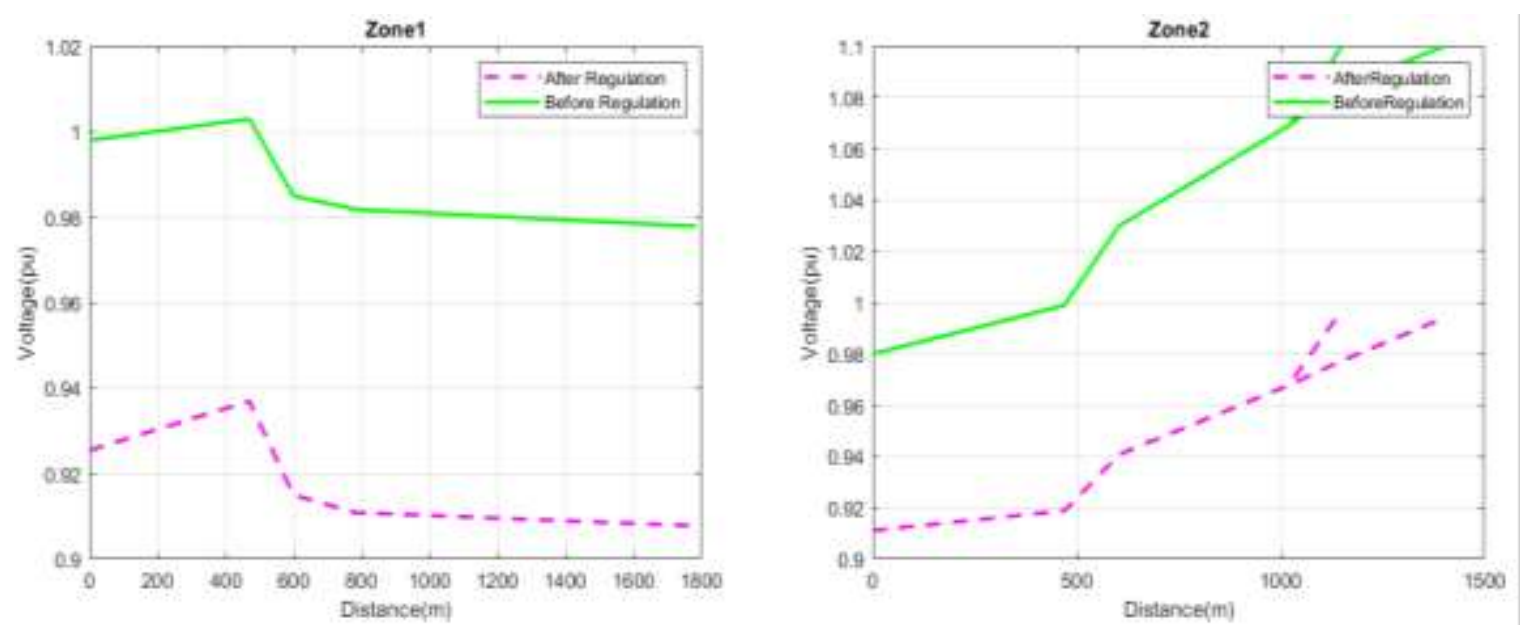

Figure 7. Voltage profile with DG implementation and control model (FFNN)
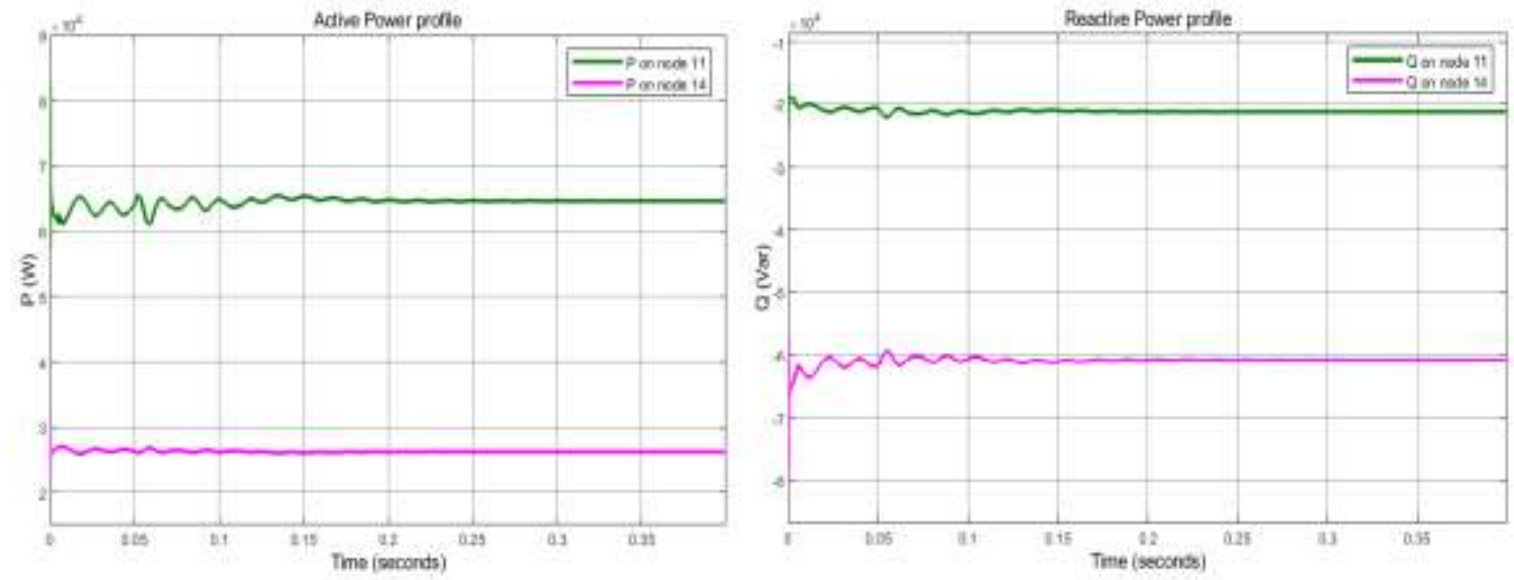

Figure 8. Power profile with DG implementation and control model (FFNN)

The training model of the two-neuron backpropagation neural network for synchronous generator is shown in Figure 12. The figure depicts that the model reaches the convergence precision at step 965. Besides, the validation performances for the synchronous generator model is shown in Figure 13. The best validation performance is attained at an MSE value of 1:0756 $\times 10-5$ and epoch 965 . Also, based on the similarity of the sets, there is no overfitting issue in the system. Similarly, the error histogram for forecasting the voltage regulation based on the developed ANN is represented in Figure 14. This plot illustrates that the majority of the errors fall in a relatively confined range of $-1: 00173$ and 0:004775, and nearly all the errors have a value of about 0:001521. Furthermore, the regressions experienced within the outputs and network targets are computed and represented. The overall prediction performances for the synchronous generator model is depicted in Figure 15 by means of plots of output and target values. The fitting performances for the considered model are good, as the data points are tightly distributed down the fitting line. Also, the obtained correlation coefficients are very close to 1 (0:9998). This indicates that the considered scheme offers a closefitting between the network targets and related outputs. Besides, the virtually linear distribution of the data points along with the output versus the target line shows that the model's prediction accuracy is good. 

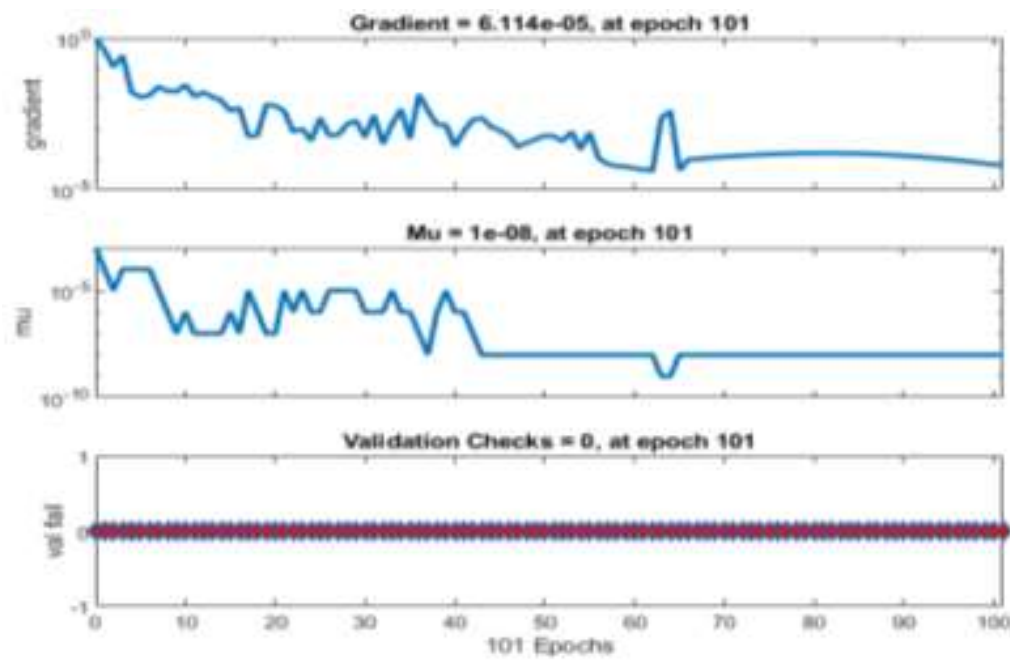

Figure 9. Training state diagram of the backpropagation neural network for solar power inverter

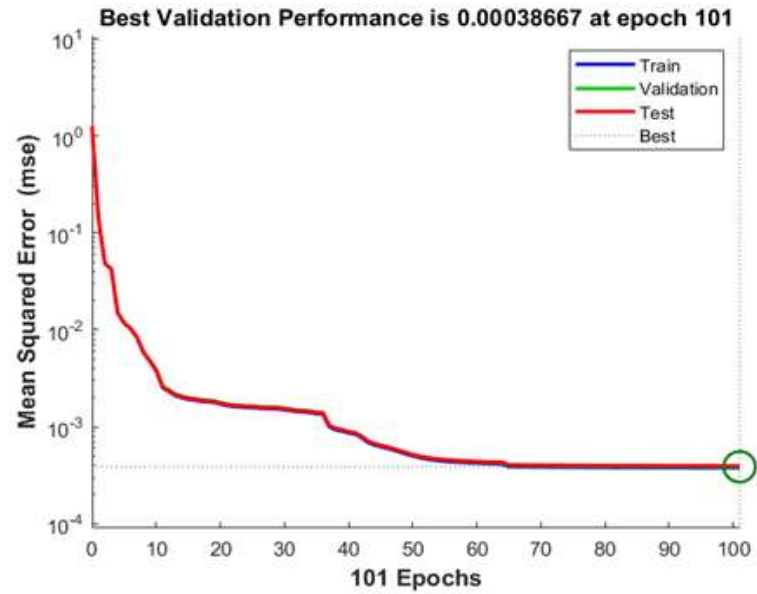

Figure 10. FFNN model validation performance for solar power inverter

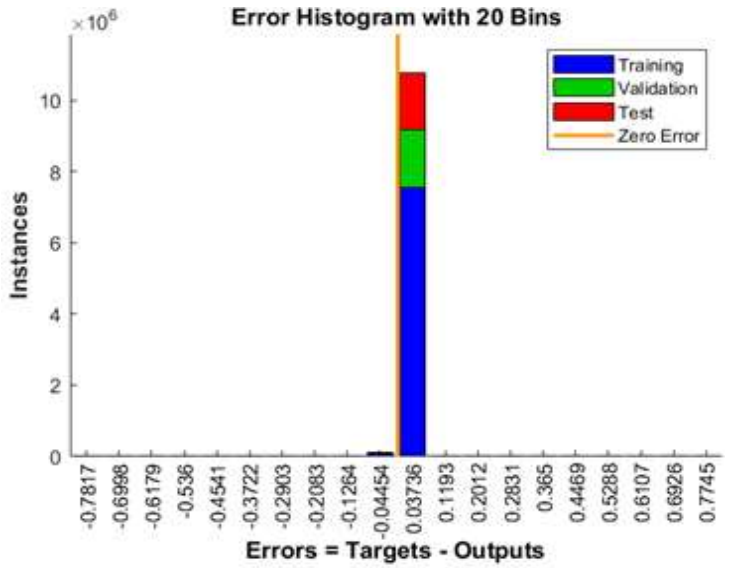

Figure 11. Error histogram for solar power inverter
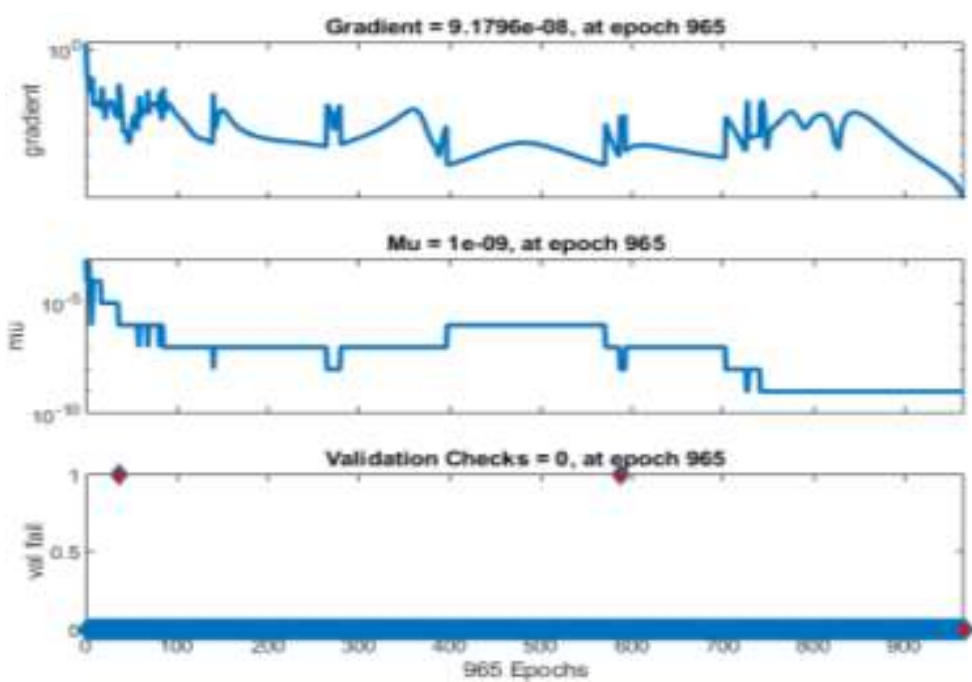

Figure 12. Training state diagram of the two-neuron backpropagation neural network for synchronous generator 


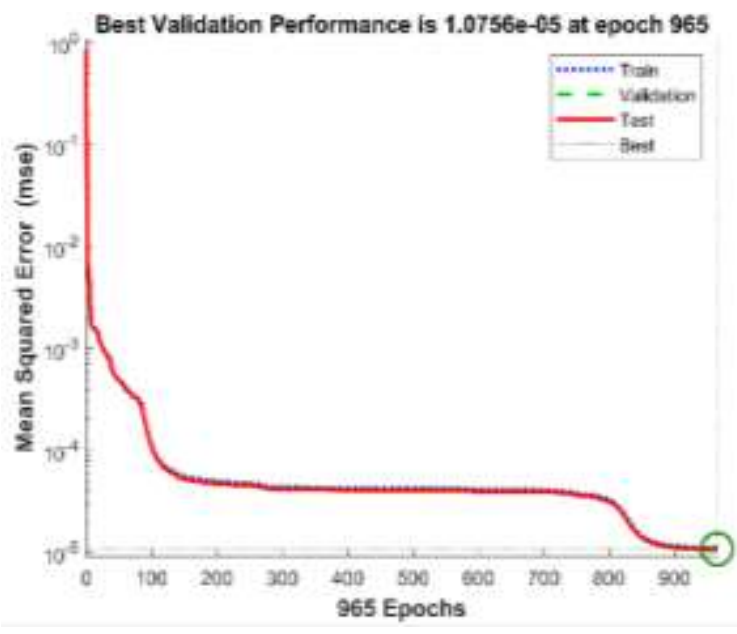

Figure 13. FFNN model validation performance for synchronous generator
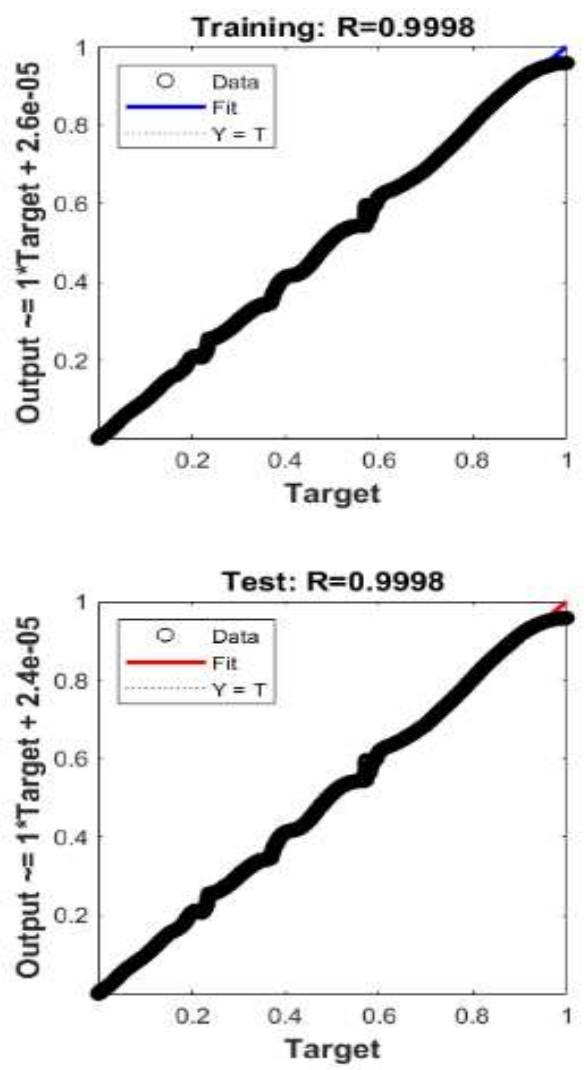

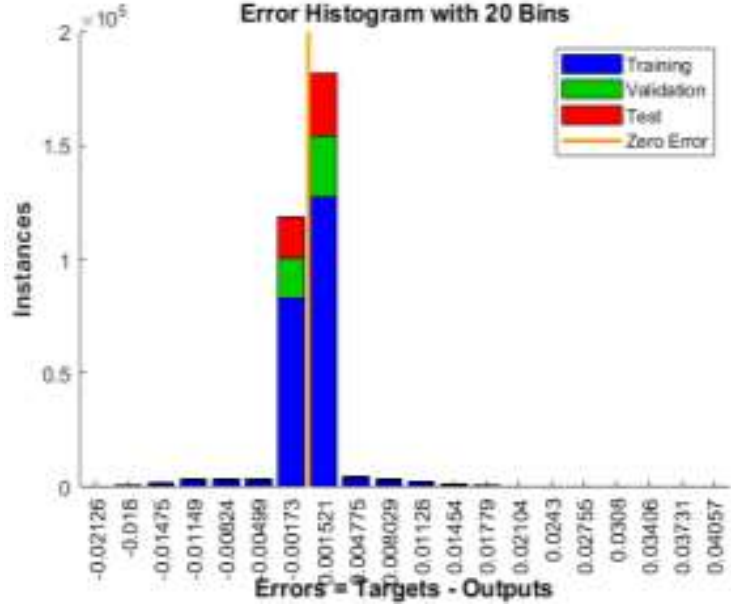

Figure 14. Error histogram for synchronous generator
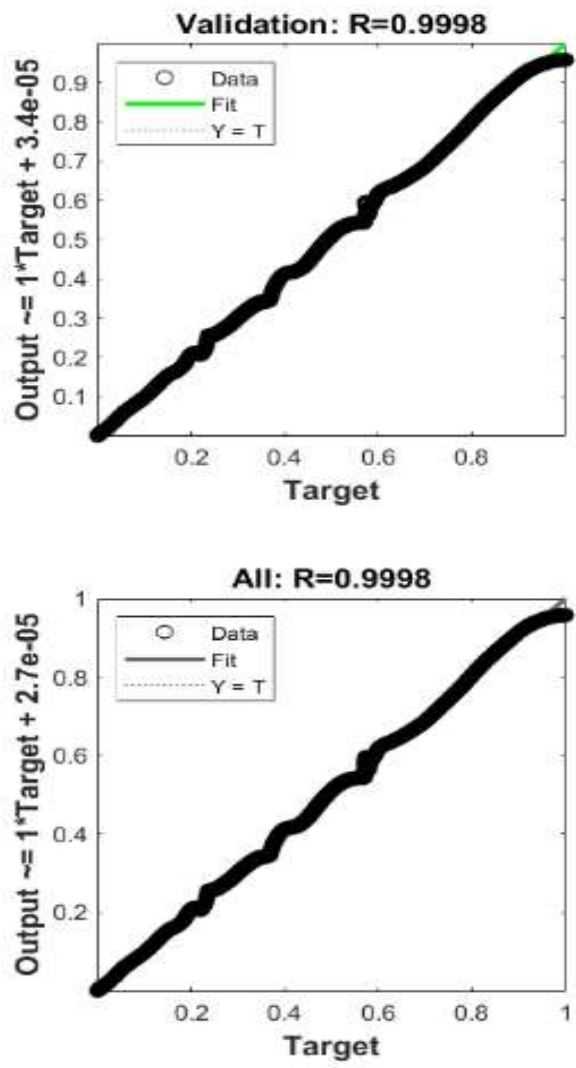

Figure 15. Regression model performance for synchronous generator. The top left blue plot depicts the training fitting, the top right green plot illustrates the validation fitting, the bottom left red plot shows the

testing fitting, and the bottom right gray plot depicts the model fitting performance for all data sets

\section{CONCLUSION}

In this paper, we have proposed a generic model for a DN with interconnected solar panel-type and wind turbine power generators. Also, we have considered and demonstrated the varied nature of the voltage under distributed generation. In this context, we have developed control models for voltage regulation. The simulation results show that developed control models can help in disturbances elimination in the DN. Moreover, we have developed and evaluated FFNN models with the backpropagation algorithm and 
leveraged the Levenberg Marquardt regularization technique to ensure effective prediction in the distributed generation-based network. Also, based on extensive simulations with MATLAB $® / \operatorname{Simulink} \AA$, we have presented the statistics values of the coefficient of determination and mean-square-error (MSE) for the considered network.

\section{REFERENCES}

[1] P. S. Revuelta, S. P. Litrán and J. P. Thomas, "8-Distributed Generation," in Active Power Line Conditioners, Eds. Design, Simulation and Implementation for Improving Power Quality San Diego, USA: Academic Press, 2016, pp. 285-322, doi: 10.1016/B978-0-12-803216-9.00008-0.

[2] O. M. Neda, "A new hybrid algorithm for solving distribution network reconfiguration under different load conditions," Indonesian Journal of Electrical Engineering and Computer Science (IJEECS), vol. 20, no. 3, pp. 1118-1127, 2020, doi: 10.11591/ijeecs.v20.i3.pp1118-1127.

[3] J. M. Guerrero et al., "Distributed Generation: Toward a New Energy Paradigm," in IEEE Industrial Electronics Magazine, vol. 4, no. 1, pp. 52-64, March 2010, doi: 10.1109/MIE.2010.935862.

[4] A. Bouakra, F. Slaoui-Hasnaoui, M. Rustom and S. Georges, "Voltage regulation of electric power network interconnected with wind energy distributed generations," 2017 IEEE Second International Conference on DC Microgrids (ICDCM), 2017, pp. 387-392, doi: 10.1109/ICDCM.2017.8001075.

[5] D. Almeida, J. Pasupuleti, J. Ekanayake and E. Karunarathne, "Mitigation of overvoltage due to high penetration of solar photovoltaics using smart inverters volt/var control", Indonesian Journal of Electrical Engineering and Computer Science (IJEECS), vol. 19, no. 3, pp. 1259-1266, 2020, doi: 10.11591/ijeecs.v19.i3.pp1259-1266.

[6] N. Hatziargyriou, H. Asano, R. Iravani and C. Marnay, "Microgrids," in IEEE Power and Energy Magazine, vol. 5, no. 4, pp. 78-94, July-Aug. 2007, doi: 10.1109/MPAE.2007.376583.

[7] J. J. Justo, F. Mwasilu, J. Lee, and J.-W. Jung, "AC-microgrids versus DC-microgrids with distributed energy resources: A review," Renewable and Sustainable Energy Reviews, vol. 24, pp. 387-405, 2013, doi: 10.1016/j.rser.2013.03.067.

[8] H. Farhangi, "The path of the smart grid," in IEEE Power and Energy Magazine, vol. 8, no. 1, pp. 18-28, JanuaryFebruary 2010, doi: 10.1109/MPE.2009.934876.

[9] H. E. Farag, E. F. El-Saadany and R. Seethapathy, "A Two Ways Communication-Based Distributed Control for Voltage Regulation in Smart Distribution Feeders," in IEEE Transactions on Smart Grid, vol. 3, no. 1, pp. 271-281, March 2012, doi: 10.1109/TSG.2011.2167355.

[10] W. El-khattam and M. A. Salama, "Impact of Distributed Generation on Voltage Profile in Deregulated Distribution System," in Proceedings of the power systems 2002 conference, impact of distributed generation, Clemson, SC, USA, 2002, pp. 13-15.

[11] J. W. Smith and D. L. Brooks, "Voltage impacts of distributed wind generation on rural distribution feeders," 2001 IEEE/PES Transmission and Distribution Conference and Exposition. Developing New Perspectives (Cat. No.01CH37294), 2001, pp. $492-497$ vol.1, doi: 10.1109/TDC.2001.971283.

[12] D. D. Li and L. Q. Niu, "Reliability analysis of electric distribution system integrated with wind power," $20083 \mathrm{rd}$ IEEE Conference on Industrial Electronics and Applications, 2008, pp. 729-733, doi: 10.1109/ICIEA.2008.4582611.

[13] J. Kondoh, H. Aki, H. Yamaguchi, A. Murata, and I. Ishii, "Study on Voltage Regulation Methods for Distribution Systems with Dispersed Generators," Electrical Engineering in Japan, vol. 124, no. 4, pp. 1432-1438, 2006, doi: 10.1002/eej.20246.

[14] T. Niknam, A. M. Ranjbar and A. R. Shirani, "Impact of distributed generation on volt/Var control in distribution networks," 2003 IEEE Bologna Power Tech Conference Proceedings,, 2003, pp. 7 pp. Vol.3-, doi: 10.1109/PTC.2003.1304390.

[15] D. Rho, H. Kita, J. Hasegawa and K. Nishiya, "A study on the optimal voltage regulation methods in power distribution systems interconnected with dispersed energy storage and generation systems," Proceedings 1995 International Conference on Energy Management and Power Delivery EMPD '95, 1995, pp. 702-707 vol.2, doi: 10.1109/EMPD.1995.500814.

[16] M. H. J. Bollen and A. Sannino, "Voltage control with inverter-based distributed generation," in IEEE Transactions on Power Delivery, vol. 20, no. 1, pp. 519-520, Jan. 2005, doi: 10.1109/TPWRD.2004.834679.

[17] D. Cortinas and P. Juston, "Assessing the impact of dispersed generation on medium voltage networks: analysis methods," PowerTech Budapest 99. Abstract Records. (Cat. No.99EX376), 1999, pp. 3-, doi: 10.1109/PTC.1999.826434.

[18] R. Caire, N. Retiere, S. Martino, C. Andrieu and N. Hadjsaid, "Impact assessment of LV distributed generation on MV distribution network," IEEE Power Engineering Society Summer Meeting,, 2002, pp. 1423-1428 vol.3, doi: 10.1109/PESS.2002.1043620.

[19] Y. Jiang, J. A. A. Qahouq and M. Orabi, "Matlab/Pspice hybrid simulation modeling of solar PV cell/module," 2011 Twenty-Sixth Annual IEEE Applied Power Electronics Conference and Exposition (APEC), 2011, pp. 1244-1250, doi: 10.1109/APEC.2011.5744752.

[20] T. Le, H. Colin, F. A. Shakarchi and T. T. Quoc, "Improved Matlab Simulink Two-diode Model of PV Module and Method of Fast Large-Scale PV System Simulation," 2018 7th International Conference on Renewable Energy Research and Applications (ICRERA), 2018, pp. 982-985, doi: 10.1109/ICRERA.2018.8566792. 
[21] N. Kacimi, S. Grouni, A. Idir and M.S. Boucherit, "New improved hybrid MPPT based on neural network-model predictive control-Kalman filter for photovoltaic system," Indonesian Journal of Electrical Engineering and Computer Science (IJEECS), vol. 20, no. 3, pp. 1230-1241, 2020, DOI: 10.11591/ijeecs.v20.i3.pp1230-1241.

[22] M. K. Dave, "Modeling of PV arrays based on datasheet," 2016 IEEE 1st International Conference on Power Electronics, Intelligent Control and Energy Systems (ICPEICES), 2016, pp. 1-4, doi: 10.1109/ICPEICES.2016.7853617.

[23] Y. Sun, J. Wang, W. Zhu, S. Yuan, Y. Hong, M. S. Mannan, and B. Wilhite, "Development of Consequent Models for Three Categories of Fire through Artificial Neural Networks," Industrial and Engineering Chemistry Research, vol. 59, no. 1, pp. 464-474, 2020, doi: 10.1021/acs.iecr.9b05032.

[24] H. Liu, "On the Levenberg-Marquardt training method for feed-forward neural networks," 2010 Sixth International Conference on Natural Computation, 2010, pp. 456-460, doi: 10.1109/ICNC.2010.5583151.

[25] C. Lv et al., "Levenberg-Marquardt Backpropagation Training of Multilayer Neural Networks for State Estimation of a Safety-Critical Cyber-Physical System," in IEEE Transactions on Industrial Informatics, vol. 14, no. 8, pp. 3436-3446, Aug. 2018, doi: 10.1109/TII.2017.2777460.

[26] I. Khan et al., "Design of Neural Network With Levenberg-Marquardt and Bayesian Regularization Backpropagation for Solving Pantograph Delay Differential Equations," in IEEE Access, vol. 8, pp. 137918137933, 2020, doi: 10.1109/ACCESS.2020.3011820.

[27] Y. Sai, R. Jinxia and L. Zhongxia, "Learning of Neural Networks Based on Weighted Mean Squares Error Function," 2009 Second International Symposium on Computational Intelligence and Design, 2009, pp. 241-244, doi: 10.1109/ISCID.2009.67. 